\title{
The Effective Length of Elastic Supporting Bars
}

\author{
Shi-lun $\mathrm{SHI}^{1, \text { a }}$, Chun-lan Liao ${ }^{2, \mathrm{~b}}$ \\ ${ }^{1}$ Construction Engineering College, Kunming University of Science and Technology, Kunming, \\ Yunnan, 650500, China \\ ${ }^{2}$ Faculty of Foreign Languages \& Cultures, Kunming University of Science and Technology, \\ Kunming, Yunnan, 650500, China \\ ashishilun1982@126.com, 'liaochunlan1984@126.com
}

Keywords: Elastic supporting bars, Stability capacity, Timoshenko elastic stability theory

\begin{abstract}
Elastic supporting bars exist in many structures, like transmission tower, truss, even frame, etc. Take transmission tower for example, the load-carrying capacity of diagonal members often controls the structure's bearing capacity. Previous experiments and engineering experiences indicated that possible unsafety exists in structures when designing diagonal members according to current codes. Based on the Timoshenko elastic stability theory, the study deduces the relations between adjacent web member's internal force and stability capacity of X-shape diagonal members and K-shape ones. The calculation formula for diagonal member's effective length factor of transmission tower was obtained, which can be reference in revising the codes.
\end{abstract}

\section{Introduction}

Diagonal members, whose stability capacity often controls the structure loading capacity, are mainly classified as X- and K-shape on transmission towers. The codes for designing such members in many countries are quite different. Take the angle steel tower as an example, American Design of Latticed Steel Transmission Structure [1] decides stability capacity by the slenderness ratio of full length divided by radius of gyration to the parallel axis; Japanese Design Criteria for Electric Transmission Line Supports [2] by the slenderness ratio of diagonal member's internode length divided by radius of gyration to the minimum axis; Chinese Technical Regulation of Design for Tower and Pole Structures of Overhead Transmission Line [3] by the slenderness ratio of internode to minimum axis or that of full length to the parallel axis respectively on the conditions of whether one is pulled and the other is pressed in the two jointed diagonal members or both are pressed. Pilot calculation shows that the results obtained from codes in different countries are quite different. At present, there is no widely-recognized and suitable standard. Therefore, it is necessary to do the theoretical and experimental researches into the mechanical behavior of diagonal members on transmission tower structures, in order to determine the loading capacity more precisely.

Transmission tower's secondary stresses and joint effects on behavior of structures are reviewed by Roy S et al [4], Al-Bermani F G A and Kitipornchai S [5], and so on. Some other scientists, such as Feng Dingguo and Yong Yudong [6], Chen Shaofan [7], Chen Y Y and Wang W [8], also review the stability capacity of transmission towers, or similar structures, like truss, but few focus on the diagonal member's stability and its influence on structure.

\section{The effective length factor of elastic supporting bars}

As an example, the schematic diagram of K-type web members with an internode is illustrated in Figure 1. When compressed web member AC buckles out of the truss plane, adjacent member BC transfers elastic support through the horizontal bar. According to the general design principles, supposing $\mathrm{AC}$ and $\mathrm{BC}$ have the same geometric parameter (length \& inertia moment), meanwhile, disregarding the effect of horizontal bar's deformation, which may cause a little error but also make the same computational formula for K-type web member and intersected diagonal member. Then, elastic support's stiffness $\alpha$ depends on the stiffness EI of BC member and its load N. Apparently, if $\mathrm{N}=0$, spring stiffness 


$$
\alpha=\frac{48 E I}{L^{3}}
$$

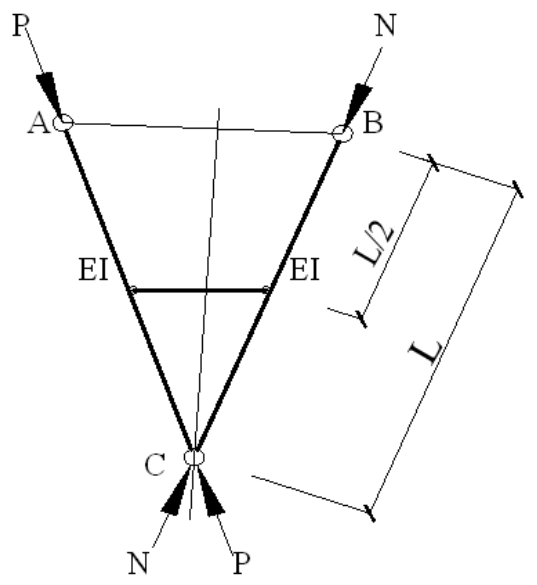

Fig. 1 The schematic diagram of web members with an internode

if $N=\eta P$ [9], expansion coefficient of deformation is used to measure the effect of adjacent member's internal force on elastic stiffness.

$$
\alpha=\frac{48 E I}{L^{3}}\left(1-\frac{N}{P}\right)=\frac{48 E I}{L^{3}}(1-\eta)
$$

where $|\eta|<1$, if the compression force of BC member $N \geq P$, no supporting force provides to AC member; if there is tensile force for BC member and $|N| \geq|P|$, it is quite a safe way not to consider the increase of bearing stiffness as $N$ increases.

According to Timoshenko elastic stability theory, the stability capacity equation of AC in Figure 1 will be

$$
-\frac{\sin k L / 2}{P k \sin k L} R \sin k L / 2+\frac{L / 2}{P L} R L / 2=\frac{R}{\alpha}
$$

setting $\beta=k L / 2, k=\sqrt{\frac{P}{E I}}$, and $P_{e}=\frac{\pi^{2} E I}{L^{2}}$, substituting into Equation (2) yields

$$
\frac{\alpha L}{P_{e}}=\frac{16 \beta^{3}}{\pi^{2}(\beta-\tan \beta)}
$$

let $\beta$ be any numerical value from $\pi / 2$ to $\pi$, the values of $\frac{P_{c r}}{P_{e}}$ and $\frac{\alpha L}{P_{e}}$ were obtained as shown in Table 1.

Table 1. The values of $\frac{P_{c r}}{P_{e}}$ and $\frac{\alpha \mathrm{L}}{P_{e}}$
\begin{tabular}{|c|c|c|}
\hline$\beta$ & $\alpha \mathrm{L} / P_{e}$ & $P_{c r} / P_{e}$ \\
\hline 1.57 & 0.00 & 1.00 \\
\hline 1.73 & 1.04 & 1.21 \\
\hline 1.88 & 2.19 & 1.44 \\
\hline 2.04 & 3.45 & 1.69 \\
\hline 2.20 & 4.82 & 1.96 \\
\hline 2.36 & 6.32 & 2.25 \\
\hline 2.51 & 7.94 & 2.56 \\
\hline 2.67 & 9.71 & 2.89 \\
\hline 2.83 & 11.62 & 3.24 \\
\hline 2.98 & 13.71 & 3.61 \\
\hline 3.14 & 16.00 & 4.00 \\
\hline
\end{tabular}


The fitting curve and formula Conducting curve are shown in Figure 2.

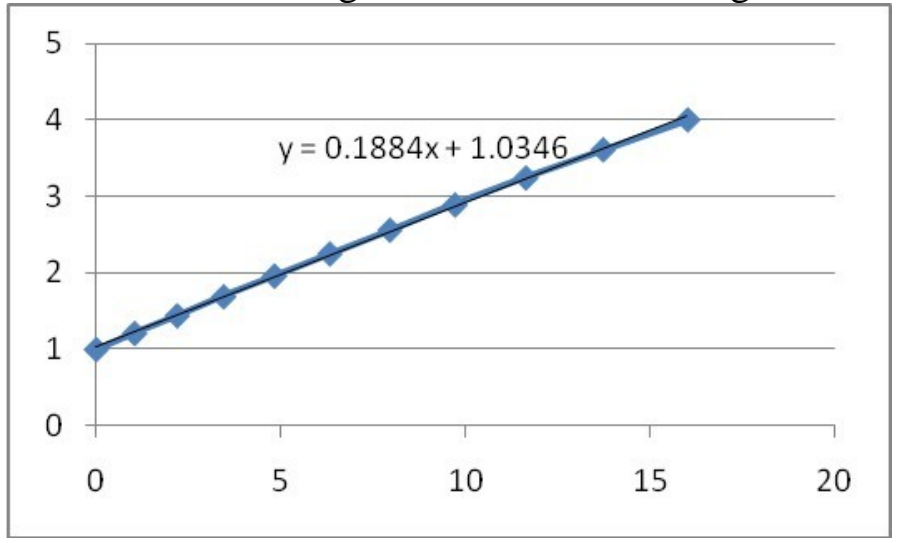

Fig. 2 The fitting curve of $\frac{P_{c r}}{P_{e}}$ and $\frac{\alpha L}{P_{e}}$

For easy application, simplify to

$\frac{P_{c r}}{P_{e}}=1+0.2 \frac{\alpha L}{P_{e}}$

Substituting Equation (1) into Equation (4) yields

$\frac{P_{c r}}{P_{e}}=1.973-0.973 \eta \approx 2-\eta$

Then the effective length factor of compressed web member is

$u=\frac{1}{\sqrt{2-\eta}}$

\section{Conclusions}

This paper deduced the effective length factor of elastically supported transmission tower diagonal member. The comparison with the Codes reached the conclusions as follows:

1) The deduction by the method discussed in this paper is simplified, coherent and theoretically reliable and logical, and the results provide certain reference value.

2) As described in DL/T 5154-2002, the effective length factor, lack of theoretical foundation, was obtained from engineering experiences and codes of other countries. The comparison reveals that the method applied in this paper concerns the relationship between the effective length factor and the internal force ratio of intersected or adjacent web members, which is more reasonable than the way in DL/T 5154-2002, because it just simply differentiate the conditions of whether these members are pulled or pressed.

3) The comparison of effective length factor respectively calculated by the method developed in this paper and that in DL/T 5154-2002 indicates that possible insecurity exists in the members designed by the latter way.

4) Method of this paper is just deduced theoretically on some hypotheses. The application into reality calls for experimental tests and verifications.

\section{Acknowledgments}

This research was supported by Applied Fundamental Research Plan Projects of Yunnan (No. 2011FZ042)

\section{References}

[1] American Society of Civil Engineers. Design of Latticed Steel Transmission Structure. (ASCE 10-97), ASCE, Reston, 2000. 
[2] Japanese Electrotechnical Committee. Design Criteria for Electric Transmission Line Supports. (JEC-127), JEC, Tokyo, 1979.

[3] Southwest Electric Power Design Institute. Technical regulation of design for tower and pole structures of overhead transmission line. (DL/T 5154-2002), China Electric Power Press, Beijing, 2002.

[4] Roy S, Fang Shujin and E. C. Rossow. Secondary stresses on transmission tower structures. Journal of Energy Engineering Vol.110 (1998), p. 157-172.

[5] Al-Bermani F G A, Kitipornchai S. Nolinear analysis of transmission towers. Engineering Structures Vol.14 (1992). p. 139-151.

[6] Feng Dingguo, Yong Yudong. Analysis of effective length of compression members in trusses. J. Xi'an Inst. of metal \& Cons. Eng. Vol.22 (1990). p. 309-318.

[7] Chen Shaofan (1996). The out-of-plane stability of truss web members and bracing requirement. Engineering Mechanics Vol.13 (1996). p. 16-25.

[8] Chen Y Y, Wang W (2003). Flexural behavior and resistance of Uni-planar KK and X tubular joints. Steel\& Composite Structures Vol.3. (2003). p. 123-140.

[9] Timoshenko. Theory of Elastic Stability. 2nd ed, New York: McGraw-Hill, 1961. 